IJMMS 29:4 (2002) 209-216

\title{
COMPACT-CALIBRES OF REGULAR AND MONOTONICALLY NORMAL SPACES
}

\author{
DAVID W. MCINTYRE
}

Received 10 January 2001 and in revised form 10 May 2001

\begin{abstract}
A topological space has calibre $\omega_{1}$ (resp., calibre $\left(\omega_{1}, \omega\right)$ ) if every point-countable (resp., point-finite) collection of nonempty open sets is countable. It has compact-calibre $\omega_{1}$ (resp., compact-calibre $\left(\omega_{1}, \omega\right)$ ) if, for every family of uncountably many nonempty open sets, there is some compact set which meets uncountably many (resp., infinitely many) of them. It has CCC (resp., DCCC) if every disjoint (resp., discrete) collection of nonempty open sets is countable. The relative strengths of these six conditions are determined for Moore spaces, regular first countable spaces, linearly-ordered spaces, and arbitrary regular spaces. It is shown that the relative strengths for spaces with point-countable bases are the same as those for Moore spaces, and the relative strengths for linearly-ordered spaces are the same as those for arbitrary monotonically normal spaces.
\end{abstract}

2000 Mathematics Subject Classification: 54A25.

1. Introduction and basic results. Let $X$ be a topological space. If every uncountable collection of nonempty open sets is point-countable (resp., point-finite) then $X$ is said to have calibre $\omega_{1}$ (resp., calibre $\left(\omega_{1}, \omega\right)$ ). If for every uncountable collection of nonempty open sets there is a compact subset of $X$ meeting uncountably many (resp., infinitely many) of the open sets then $X$ is said to have compact-calibre $\omega_{1}$ (resp., compact-calibre $\left(\omega_{1}, \omega\right)$ ). If every disjoint (resp., discrete) collection of nonempty open sets is countable, then $X$ is said to have the countable chain condition (CCC) (resp., the discrete countable chain condition (DCCC)). Clearly, calibre $\omega_{1}$ implies calibre $\left(\omega_{1}, \omega\right)$, calibre $\left(\omega_{1}, \omega\right)$ implies CCC, and CCC implies DCCC. Also, calibre $\omega_{1}$ implies compact-calibre $\omega_{1}$, and either compact-calibre $\omega_{1}$ or calibre $\left(\omega_{1}, \omega\right)$ implies compact-calibre $\left(\omega_{1}, \omega\right)$.

Proposition 1.1. Every space with compact-calibre $\left(\omega_{1}, \omega\right)$ has DCCC.

Proof. Let $(X, \mathscr{T})$ be a space which has a discrete family $\left\{U_{\alpha}: \alpha \in \omega_{1}\right\}$ of nonempty open sets. Suppose $K \subseteq X$ meets $U_{\alpha}$ for infinitely many $\alpha \in \omega_{1}$. Put $\Lambda=\left\{\alpha \in \omega_{1}\right.$ : $\left.K \cap U_{\alpha} \neq \varnothing\right\}$. Then $\left\{K \cap \overline{U_{\alpha}}: \alpha \in \Lambda\right\}$ is an infinite discrete collection of closed subsets of $K$, so $K$ is not compact.

These simple relations are summarized in Figure 1.1.

In general, none of the implications in Figure 1.1 can be reversed, and no other implications hold between the conditions. However, in the presence of extra assumptions about the space, some of these conditions become equivalent. For example, all of these conditions hold in separable spaces, so since DCCC metrizable spaces are separable, these conditions are all equivalent for metrizable spaces. Similarly, if Souslin's 


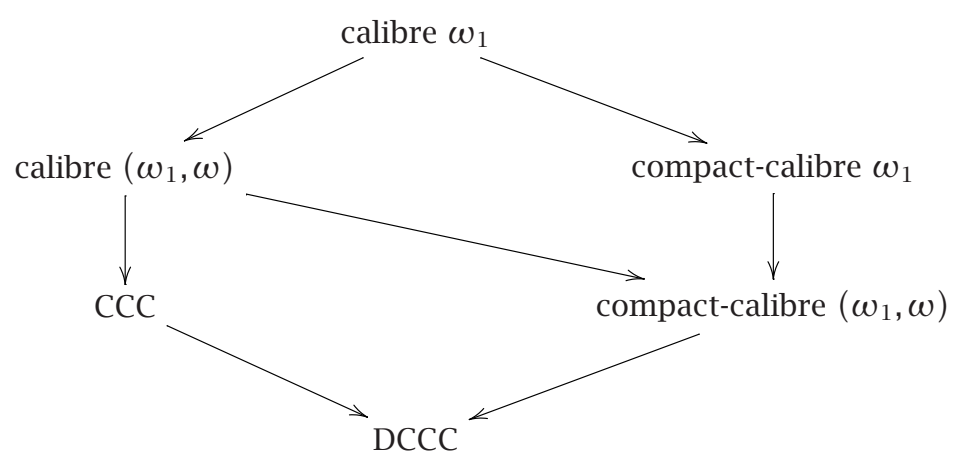

FIGURE 1.1. Relative strengths of the conditions for arbitrary spaces.

Hypothesis (SH) holds then any CCC linearly-ordered space is separable and so CCC implies each of the other conditions under that assumption. We will discuss what happens when we weaken metrizability, and what happens when SH fails.

The classic weakening of metrizability is to Moore spaces. In the next section, we will see that for Moore spaces calibre $\omega_{1}$, calibre $\left(\omega_{1}, \omega\right)$, CCC, and DCCC are distinct, while compact-calibre $\omega_{1}$ is equivalent to calibre $\omega_{1}$ and compact-calibre $\left(\omega_{1}, \omega\right)$ is equivalent to DCCC. For normal Moore spaces, DCCC is equivalent to CCC, which may or may not be equivalent to calibre $\omega_{1}$.

In Section 3, we will see that for linearly-ordered spaces calibre $\omega_{1}$, compact-calibre $\omega_{1}$, compact-calibre $\left(\omega_{1}, \omega\right)$, and DCCC are distinct, while calibre $\left(\omega_{1}, \omega\right)$ and CCC are equivalent. Depending on whether or not SH holds, CCC is either equivalent to calibre $\omega_{1}$ or does not even imply compact-calibre $\omega_{1}$. As so often occurs, we can replace "linearly ordered" by "monotonically normal" in these statements.

In Section 4, we will show that for arbitrary regular spaces, no implications, other than those in Figure 1.1, hold between the conditions.

2. Moore spaces. First we will show that, for Moore spaces, compact-calibre $\omega_{1}$ is equivalent to calibre $\omega_{1}$, and DCCC is equivalent to compact-calibre $\left(\omega_{1}, \omega\right)$.

Proposition 2.1. Any Moore space with compact-calibre $\omega_{1}$ has calibre $\omega_{1}$.

Proof. Let $(X, \mathscr{T})$ be a Moore space with compact-calibre $\omega_{1}$, and let $\left\{U_{\alpha}: \alpha \in \omega_{1}\right\}$ be a collection of nonempty open subsets of $X$. Let $K$ be a compact subset of $X$ and $\Gamma$ an uncountable subset of $\omega_{1}$ such that, for every $\alpha \in \Gamma, K \cap U_{\alpha} \neq \varnothing$. Then $K$ is a compact Moore space, and hence separable and metrizable. Moreover, the family $\left\{U_{\alpha} \cap K: \alpha \in \Gamma\right\}$ is an uncountable family of nonempty open subsets of $K$, and hence not point-countable. Thus, the original family is not point-countable.

Proposition 2.2. Let $(X, \mathcal{T})$ be a regular first countable space with DCCC. Then $(X, \mathcal{T})$ has compact-calibre $\left(\omega_{1}, \omega\right)$. 
PROoF. In a regular DCCC space, any locally finite collection of nonempty open sets is countable [19]. Thus, if $\left\{U_{\alpha}: \alpha \in \omega_{1}\right\}$ is a collection of nonempty open subsets of $X$, then there is some $x \in X$ such that every neighbourhood of $x$ meets $U_{\alpha}$ for infinitely many $\alpha \in \omega_{1}$. Let $\left\{B_{n}(x): n \in \omega\right\}$ be a local basis at $x$ such that, for every $n \in \omega, B_{n+1}(x) \subseteq B_{n}(x)$. We define sequences $\left(\alpha_{n}\right)_{n \in \omega}$ in $\omega_{1}$ and $\left(x_{n}\right)_{n \in \omega}$ in $X$ as follows: pick $\alpha_{0} \in \omega_{1}$ such that $B_{0}(x) \cap U_{\alpha_{0}} \neq \varnothing$, and pick $x_{0} \in B_{0}(x) \cap U_{\alpha_{0}}$. Now let $n \in \omega$, and suppose that we have already chosen $\alpha_{m} \in \omega_{1}$ and $x_{m} \in X$ for every $m<n$. Choose $\alpha_{n}$ such that, for every $m<n, \alpha_{n} \neq \alpha_{m}$, and $B_{n}(x) \cap U_{\alpha_{n}} \neq \varnothing$, and choose $x_{n} \in B_{n}(x) \cap U_{\alpha_{n}}$. Put $K=\{x\} \cup\left\{x_{n}: n \in \omega\right\}$. Then $K$ is a compact subset of $X$ which meets $U_{\alpha}$ for infinitely many $\alpha \in \omega_{1}$. Thus, $(X, \mathcal{T})$ has compact-calibre $\left(\omega_{1}, \omega\right)$.

The hypothesis that $X$ is first countable is needed in Proposition 2.2, as is shown by Example 4.1. On the other hand, the hypothesis of regularity is only needed in order to invoke Wiscamb's result that DCCC spaces have what might be termed the "locally finite countable chain condition" or LFCCC: every locally finite collection of nonempty open sets is countable. As far as the author is aware, no other results have been published on this property. The most natural question to ask is the following.

QUESTION 2.3. For which classes of spaces does DCCC imply LFCCC?

The author knows of a $T_{1}$ space with DCCC but not LFCCC, but no Hausdorff example.

Let $(X, \mathscr{T})$ be a regular first countable space. For each $x \in X$, fix a local basis $\{B(x, n): n \in \omega\}$ at $x$ such that, for each $n \in \omega, \overline{B(x, n+1)} \subseteq B(x, n)$. Let $M=$ $X \times{ }^{<\omega} \omega$, and for $p=\langle x, f\rangle \in M$ and $n \in \omega$ define

$$
G_{n}(p)=\{p\} \cup\left\{\left\langle y, f^{-} g\right\rangle:(g \neq \varnothing) \wedge(g(0) \geq n) \wedge(y \in B(x, n+g(0)))\right\},
$$

where $f^{-} g$ denotes the concatenation of the functions $f$ and $g$. Then $\left\{G_{n}(p): p \in\right.$ $M, n \in \omega\}$ forms a basis for a topology $\mathscr{Y}$ on $M$. The space $(M, \mathscr{Y})$ is a Moore space, called the Reed space over $X$. A number of chain conditions are preserved between $(X, \mathscr{T})$ and $(M, \mathscr{S})$, including DCCC, CCC, calibre $\left(\omega_{1}, \omega\right)$, calibre $\omega_{1}$, and separability (see Reed [11] and McIntyre [5]). The construction was introduced in the case where $X$ is $\omega_{1}$, with its order topology, in order to construct a Moore space with DCCC but without CCC (see Reed [10]). A variation of this construction was used in [12] to construct a Moore space with calibre $\left(\omega_{1}, \omega\right)$ but without calibre $\omega_{1}$. The set of finite subsets of $\mathbb{R}$, with the Pixley-Roy topology, is a Moore space with CCC but without calibre $\left(\omega_{1}, \omega\right)$ [5]. Thus the relationships between the six conditions are as shown in Figure 2.1.

In proving that Moore spaces with compact-calibre $\omega_{1}$ have calibre $\omega_{1}$, what we needed was the fact that compact subsets are metrizable. This is not true in regular first-countable spaces, as will be shown by Example 3.3. However, it does hold for spaces with a point-countable base [7], and thus spaces with compact-calibre $\left(\omega_{1}, \omega\right)$ and a point-countable base have calibre $\omega_{1}$. The Moore space with calibre $\left(\omega_{1}, \omega\right)$ but without calibre $\omega_{1}$ in [12] and the Moore space with CCC but without calibre $\left(\omega_{1}, \omega\right)$ (Pixley-Roy space) both have point-countable bases. Finally, we consider DCCC 


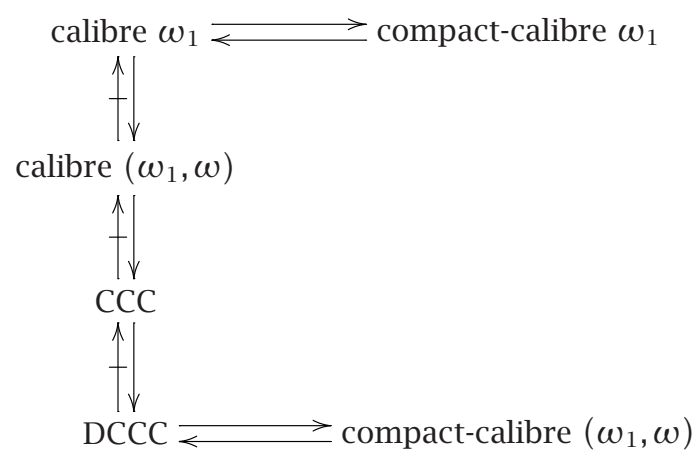

FIGURE 2.1. Relative strengths of the conditions for Moore spaces and for spaces with point-countable bases.

versus CCC for spaces with a point-countable base. Shakhmatov has shown that there exist pseudocompact (and hence DCCC) zero-dimensional spaces with point-countable bases containing closed discrete subsets of arbitrary cardinality [13]. In particular, there is a pseudocompact space with a point-countable base with cardinality greater than $2^{\omega}$, whereas any CCC first-countable space has cardinality at most $2^{\omega}$. Thus, the relative strengths of the conditions for spaces with a point-countable base are the same as the strengths for Moore spaces.

Metrizability can be thought of as having two factors, developability and separation. What happens when we add some extra separation? In normal Moore spaces, DCCC and CCC are equivalent. This follows from results in [10], where Reed shows that a Moore space has CCC if and only if it has DCCC and is wd-normal (in other words, if for every open set $U$ there is a sequence $\left(D_{n}\right)_{n \in \omega}$ of open sets such that for each $n \in \omega, \overline{D_{n}} \subseteq U$ and $\left.U \subseteq \overline{U_{n \in \omega} D_{n}}\right)$. Since normal Moore spaces are perfectly normal, they are wd-normal.

We do not require the full strength of developability for normal DCCC spaces to have CCC. Recall that a $\sigma$-space is a space with a $\sigma$-discrete network, and that Moore spaces are $\sigma$-spaces (see [1, Section 4]). Suppose that a normal $\sigma$-space $X$ has an uncountable disjoint collection of nonempty open sets. By the $\sigma$-space property, these can be shrunk to a $\sigma$-discrete collection of closed nonempty sets which are separated by the original open sets. Since in a normal space any discrete family of closed sets, which can be separated by disjoint open sets, can be separated by a discrete family of open sets, we can find an uncountable discrete family of open sets in $X$. Thus, if $X$ is a normal $\sigma$-space with DCCC, then $X$ has CCC.

In particular, stratifiable DCCC spaces have CCC.

What about the other conditions for normal Moore spaces? Przymusinski and Tall showed in [9] that it is consistent and independent that CCC normal Moore spaces are separable. Indeed, they show that under Martin's Axiom together with the negation of the Continuum Hypothesis (MA $+\neg \mathrm{CH}$ ), if $S \subseteq \mathbb{R}$ with $\omega<|S|<2^{\omega}$ then the set of finite subsets of $S$ with the Pixley-Roy topology is a metacompact normal nonseparable CCC 
Moore space (see [17] for details). This space does not have calibre $\left(\omega_{1}, \omega\right)$. On the other hand, Shapirovskij proved in [15] that if $2^{\omega}<2^{\omega_{1}}$ then every CCC normal Moore space is metrizable.

The author is unaware of any (consistent) example of a normal Moore space with calibre $\left(\omega_{1}, \omega\right)$ but without calibre $\omega_{1}$.

3. Linearly-ordered spaces and monotonically normal spaces. We have seen that in Moore spaces, DCCC and the calibres are all distinct, whereas the compact-calibres are all equivalent to one of those conditions. We will see that in linearly-ordered spaces the situation is reversed: the calibres collapse together while the compact-calibres become distinct.

EXAMPLE 3.1. A linearly-ordered space with DCCC but without compact-calibre $\left(\omega_{1}, \omega\right)$.

Let $X=\left(\omega_{1} \times(0,1)\right) \cup\left\{\left\langle\omega_{1}, 0\right\rangle\right\}$, ordered lexicographically, that is, by defining

$$
\langle\alpha, x\rangle\langle\langle\beta, y\rangle \Longleftrightarrow(\alpha<\beta) \vee((\alpha=\beta) \wedge(x<y))
$$

Suppose $\left\{U_{\alpha}: \alpha \in \omega_{1}\right\}$ is a discrete collection of nonempty open sets. For each $\alpha \in$ $\omega_{1}$, pick some $\beta_{\alpha} \in \omega_{1}$ and some $x_{\alpha} \in(0,1)$ such that $\left\langle\beta_{\alpha}, y_{\alpha}\right\rangle \in U_{\alpha}$ (note that we can do this, because $\left\langle\omega_{1}, 0\right\rangle$ is not an isolated point of $X$ ). By discreteness, there is a neighbourhood of $\left\langle\omega_{1}, 0\right\rangle$ containing at most one $\left\langle\beta_{\alpha}, y_{\alpha}\right\rangle$, so $\left\{\beta_{\alpha}: \alpha \in \omega_{1}\right\}$ is bounded. But then, there is some $\gamma$ such that $\beta_{\alpha}=\gamma$ for uncountably many $\alpha \in \omega_{1}$, so by separability of $\{\gamma\} \times(0,1)$ the collection is not disjoint and hence is not discrete. Thus, $X$ has DCCC.

On the other hand, for each $\alpha \in \omega_{1}$, put $V_{\alpha}=\{\alpha\} \times(0,1)$. Then each $V_{\alpha}$ is open in $X$. Let $K$ be a subset of $X$, and let $\Gamma=\left\{\alpha \in \omega_{1}: K \cap V_{\alpha} \neq \varnothing\right\}$. If $\Gamma$ is infinite, then let $\Lambda$ be a countably infinite subset of $\Gamma$. For each $\alpha \in \Lambda$, pick $x_{\alpha} \in(0,1)$ such that $\left\langle\alpha, x_{\alpha}\right\rangle \in K$. Then $\left\{\left\langle\alpha, x_{\alpha}\right\rangle: \alpha \in \Lambda\right\}$ is an infinite closed discrete subset of $K$, so $K$ cannot be compact. Thus, $X$ does not have compact-calibre $\left(\omega_{1}, \omega\right)$.

A linearly-ordered space with CCC is hereditarily Lindelöf [4], and hence has calibre $\left(\omega_{1}, \omega\right)$ [5]. If Souslin's hypothesis holds, then it is also separable, and hence has calibre $\omega_{1}$. However, the square of any Souslin space (i.e., any linearly-ordered CCC nonseparable space) does not have CCC [3], whereas calibre $\omega_{1}$ is preserved by products [14]. Thus, no Souslin space has calibre $\omega_{1}$.

EXAMPLE 3.2. If Souslin's hypothesis fails, then there is a linearly-ordered space with CCC but without compact-calibre $\omega_{1}$.

If there is a Souslin space, then there is one in which the interval between any two of its elements is nonseparable. One can easily adapt the proofs of the corresponding theorems about the real line to show that such a space $S$ has exactly $2^{\omega}$ many open sets, and each closed uncountable set has cardinality $2^{\omega}$, and to deduce that there exist disjoint subsets $X$ and $Y$ of $S$ such that, if $K$ is an uncountable subset of $S$, then $\bar{K} \cap X \neq \varnothing \neq \bar{K} \cap Y$. Put $Z=S \backslash Y$. Then $Z$ is (order) dense in $S$, so it is a Souslin space. However, any uncountable subset $K$ of $Z$ is not closed in $S$, and is hence not compact. 
Thus, $Z$ does not have any uncountable compact sets, and it does not have calibre $\omega_{1}$, so it does not have compact-calibre $\omega_{1}$.

EXAMPLE 3.3. A first countable linearly-ordered space with compact-calibre $\omega_{1}$ but without CCC.

Let $X=[0,1] \times[0,1]$, ordered lexicographically. This space is compact (and hence has compact-calibre $\omega_{1}$ ), and is first countable, but does not have CCC (see Steen and Seebach [16]).

It is a standard observation that most results about linearly-ordered spaces are "really" results about monotonically normal spaces. The observation applies in this case, as the results we have just stated for linearly-ordered spaces all hold for monotonically normal spaces. Monotonically normal spaces with CCC are hereditarily Lindelöf [8], Souslin's hypothesis is equivalent to the assertion that every CCC monotonically normal space is separable [18], and the square of any CCC nonseparable monotonically normal space does not have CCC (see Proposition 3.4).

We take the following definition of monotone normality: a $T_{1}$ space $(X, \mathscr{T})$ is monotonically normal, provided that there is a function $V: X \times \mathscr{T} \rightarrow \mathscr{T}$ satisfying the following conditions:

(1) if $x \in U \subseteq U^{\prime}$ with $U, U^{\prime} \in \mathcal{T}$ then $x \in V(x, U) \subseteq V\left(x, U^{\prime}\right) \subseteq U^{\prime}$; and

(2) if $x, y \in X$ with $x \neq y$ then $V(x, X \backslash\{y\}) \cap V(y, X \backslash\{x\})=\varnothing$.

Such a function $V$ is called an MN operator. All linearly-ordered spaces are monotonically normal [2].

Proposition 3.4. Let X be a CCC nonseparable monotonically normal space. Then $X^{2}$ does not have CCC.

Proof. Let $V$ be an MN operator on $X$. Let $W$ be the set of isolated points of $X$. Since $X$ has CCC, $W$ is countable. We now define, for $\alpha \in \omega_{1}$, points $a_{\alpha}, b_{\alpha}$, and $c_{\alpha}$ as follows. Suppose $\alpha \in \omega_{1}$, and $a_{\beta}, b_{\beta}$, and $c_{\beta}$ have been chosen for all $\beta<\alpha$. Let $S_{\alpha}=W \cup\left\{a_{\beta}: \beta<\alpha\right\} \cup\left\{b_{\beta}: \beta<\alpha\right\}$. Since $S_{\alpha}$ is countable, it is not dense, so there is some point $c_{\alpha} \in X \backslash \overline{S_{\alpha}}$. Choose distinct points $a_{\alpha}, b_{\alpha} \in V\left(c_{\alpha}, X \backslash \overline{S_{\alpha}}\right)$, and define

$$
\begin{aligned}
P_{\alpha} & =V\left(c_{\alpha}, X \backslash \overline{S_{\alpha}}\right), \\
U_{\alpha} & =V\left(a_{\alpha}, V\left(a_{\alpha}, P_{\alpha} \backslash\left\{b_{\alpha}\right\}\right)\right), \\
V_{\alpha} & =V\left(b_{\alpha}, V\left(b_{\alpha}, P_{\alpha} \backslash\left\{a_{\alpha}\right\}\right)\right), \\
W_{\alpha} & =U_{\alpha} \times V_{\alpha} .
\end{aligned}
$$

Notice that, for any open set $U, x \in U$ and $z \in X$, if $V(z, X \backslash\{x\}) \cap V(x, U) \neq \varnothing$ then $z \in U$. Thus if $\beta<\alpha$ and $P_{\alpha} \cap U_{\beta} \neq \varnothing$ then $c_{\alpha} \in V\left(a_{\beta}, P_{\beta} \backslash\left\{b_{\beta}\right\}\right)$, and if $P_{\alpha} \cap V_{\beta} \neq \varnothing$ then $c_{\alpha} \in V\left(b_{\beta}, P_{\beta} \backslash\left\{a_{\beta}\right\}\right)$. Since $V\left(a_{\beta}, P_{\beta} \backslash\left\{b_{\beta}\right\}\right)$ and $V\left(b_{\beta}, P_{\beta} \backslash\left\{a_{\beta}\right\}\right)$ are disjoint, $P_{\alpha}$ can meet at most one of $U_{\beta}$ and $V_{\beta}$. Hence, since $U_{\alpha}, V_{\alpha} \subseteq P_{\alpha}$, either $U_{\alpha} \cap U_{\beta}=\varnothing$ or $V_{\alpha} \cap V_{\beta}=\varnothing$ (or both). In either case, $W_{\alpha} \cap W_{\beta}=\varnothing$, so $\left\{W_{\alpha}: \alpha \in \omega_{1}\right\}$ is an uncountable disjoint collection of nonempty open subsets of $X^{2}$. Hence $X^{2}$ does not have CCC.

We summarize these results in Figure 3.1. In this figure, dotted arrows indicate that the implication is consistent with, and independent of, ZFC. 


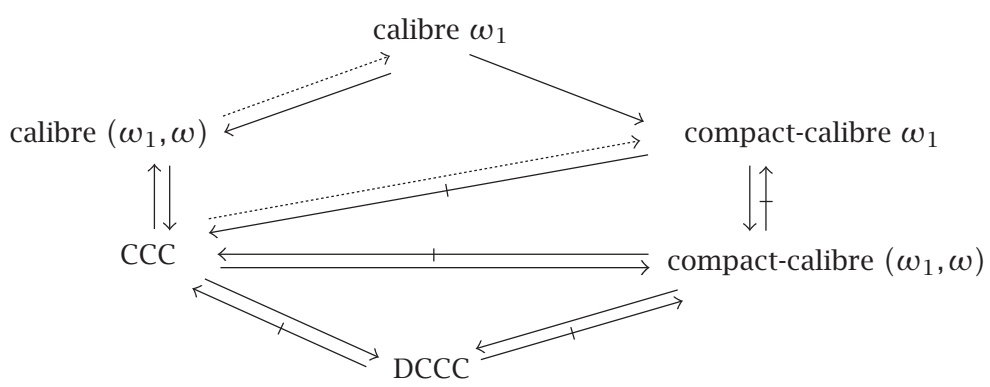

FIGURE 3.1. Relative strengths of the conditions for linearly-ordered and for monotonically normal spaces.

4. Other classes of spaces. The results above determine all the relations between the six conditions for linearly-ordered spaces, monotonically normal spaces, and Moore spaces. What about arbitrary regular spaces? Of course, all the implications in Figure 1.1 hold. If we consider all the other possible implications, we have mentioned a counterexample, either in the class of linearly-ordered spaces or in the class of Moore spaces, for every implication except that from CCC to compact-calibre $\left(\omega_{1}, \omega\right)$. This implication holds for first countable regular spaces, but does not hold for arbitrary regular spaces, as shown by Example 4.1 below. Thus, the implications in Figure 1.1 are the only ones that hold for arbitrary regular spaces.

EXAMPLE 4.1. A CCC regular space without compact-calibre $\left(\omega_{1}, \omega\right)$.

Let $\tilde{\mathbb{R}}$ denote the set of real numbers with the topology whose base consists of all sets of the form $U \backslash C$, where $U$ is an open interval and $C$ consists of the points of a nontrivial convergent sequence (without its limit). Let $X$ denote the set of finite subsets of $\tilde{\mathbb{R}}$, with the Pixley-Roy topology. Then $X$ has CCC but does not have any infinite compact subsets. Since $X$ does not have calibre $\left(\omega_{1}, \omega\right)$, it does not have compact-calibre $\left(\omega_{1}, \omega\right)$. See [6] for details.

If regularity alone is not enough to make any of these conditions equivalent, then what more is needed? First-countability is enough to make DCCC equivalent to compact-calibre $\left(\omega_{1}, \omega\right)$, but it is not enough to make DCCC equivalent to CCC nor to make compact-calibre $\omega_{1}$ equivalent to calibre $\omega_{1}$, even if we strengthen regularity to monotone normality. The first of these is shown by $\omega_{1}$ with the order topology, the second by Example 3.3. The examples in Section 2 show that CCC, calibre $\left(\omega_{1}, \omega\right)$, and calibre $\omega_{1}$ are distinct for first-countable regular spaces.

ACKNOWLEDGEMENTs. I would like to thank Paul Gartside and Mike Reed for a number of helpful comments and suggestions.

\section{REFERENCES}

[1] G. Gruenhage, Generalized metric spaces, Handbook of Set-Theoretic Topology, NorthHolland, Amsterdam, 1984, pp. 423-501.

[2] R. W. Heath, D. J. Lutzer, and P. L. Zenor, Monotonically normal spaces, Trans. Amer. Math. Soc. 178 (1973), 481-493. 
[3] G. Kurepa, La condition de Souslin et une propriété caractéristique des nombres réels, C. R. Acad. Sci. Paris 231 (1950), 1113-1114 (French).

[4] D. J. Lutzer and H. R. Bennett, Separability, the countable chain condition and the Lindelöf property in linearly orderable spaces, Proc. Amer. Math. Soc. 23 (1969), 664-667.

[5] D. W. McIntyre, Chain conditions and calibres in Moore spaces, Topology Proc. 15 (1990), 121-127.

[6] _ A regular countable chain condition space without compact-caliber $\left(\omega_{1}, \omega\right)$, Papers on General Topology and Applications (Madison, WI, 1991), New York Academy of Sciences, New York, 1993, pp. 269-272.

[7] A. Mishchenko, Spaces with a point-countable base, Soviet Math. Dokl. 3 (1962), 855-858.

[8] A. J. Ostaszewski, Monotone normality and $G_{\delta}$-diagonals in the class of inductively generated spaces, Topology, Vol. II (Proc. Fourth Colloq., Budapest, 1978), Colloq. Math. Soc. Janos Bolyai, vol. 23, North-Holland, Amsterdam, 1980, pp. 905-930.

[9] T. C. Przymusiǹski and F. D. Tall, The undecidability of the existence of a non-separable normal Moore space satisfying the countable chain condition, Fundam. Math. 85 (1974), 291-297.

[10] G. M. Reed, On chain conditions in Moore spaces, General Topology Appl. 4 (1974), 255267.

[11] _ On continuous images of Moore spaces, Canad. J. Math. 26 (1974), 1475-1479.

[12] G. M. Reed and D. W. McIntyre, A Moore space with calibre $\left(\omega_{1}, \omega\right)$ but without calibre $\omega_{1}$, Topology Appl. 44 (1992), no. 1-3, 325-329.

[13] D. B. Shakhmatov, On pseudocompact spaces with point-countable base, Soviet Math. Dokl. 30 (1984), 747-751.

[14] N. A. Shanin, On intersection of open subsets in the product of topological spaces, C. R. (Doklady) Acad. Sci. URSS (N.S.) 53 (1946), 499-501.

[15] B. Shapirovskij, On separability and metrizability of spaces with Souslin's condition, Soviet Math. Dokl. 13 (1972), 1633-1638.

[16] L. A. Steen and J. A. Seebach Jr., Counterexamples in Topology, Holt, New York, 1970.

[17] F. D. Tall, Normality versus collectionwise normality, Handbook of Set-Theoretic Topology, North-Holland, Amsterdam, 1984, pp. 685-732.

[18] S. W. Williams and H. X. Zhou, Strong versions of normality, General Topology and Applications (Staten Island, NY, 1989), Lecture Notes in Pure and Appl. Math., vol. 134, Dekker, New York, 1991, pp. 379-389.

[19] M. R. Wiscamb, The discrete countable chain condition, Proc. Amer. Math. Soc. 23 (1969), 608-612.

DAVid W. Mcintyre: Department of MAthematics, University of Auckland, Private

BAG 92019, AUCKLAND, NEW ZEALAND

E-mail address: mcintyre@math. auck1and.ac.nz 


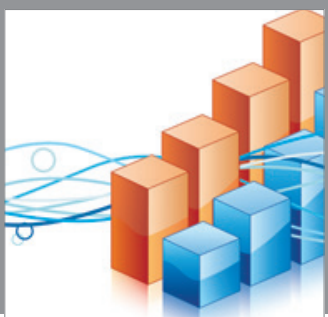

Advances in

Operations Research

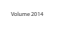

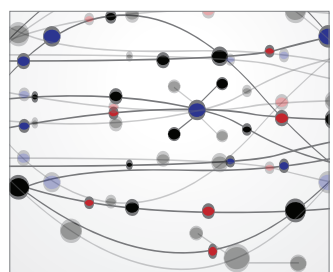

\section{The Scientific} World Journal
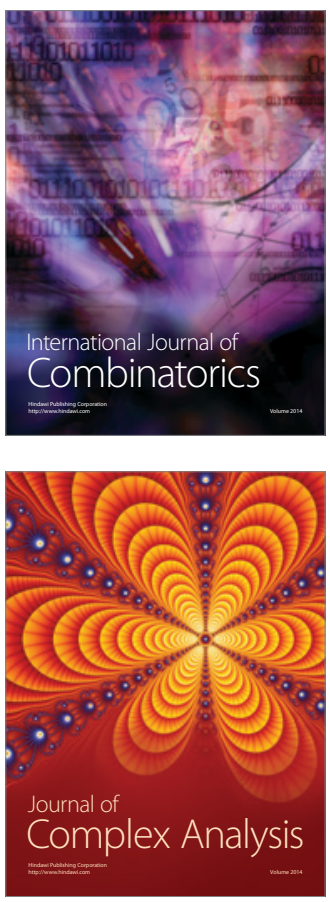

International Journal of

Mathematics and

Mathematical

Sciences
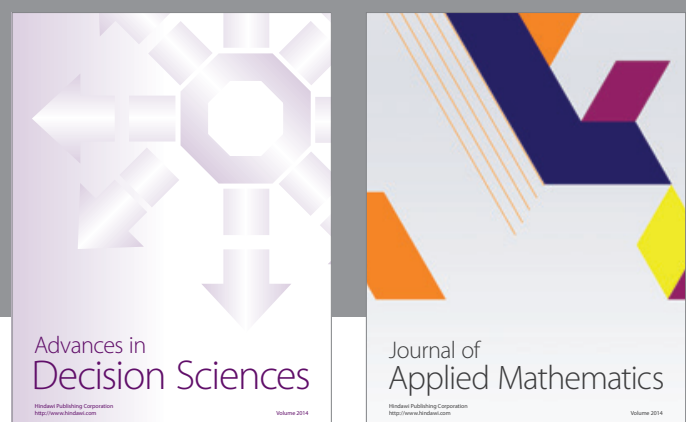

Journal of

Applied Mathematics
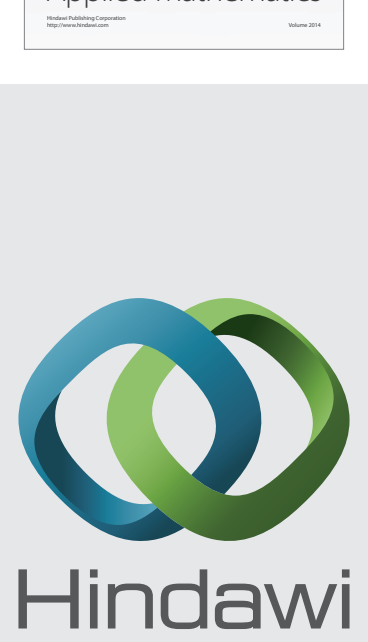

Submit your manuscripts at http://www.hindawi.com
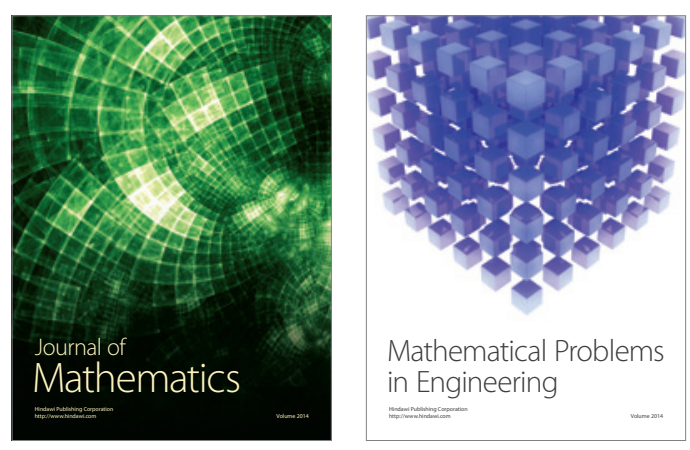

Mathematical Problems in Engineering
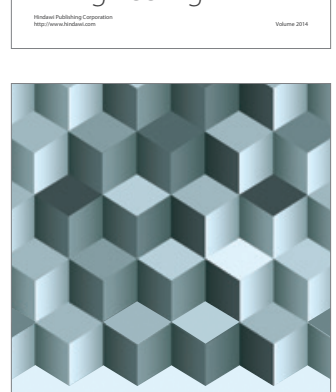

Journal of

Function Spaces
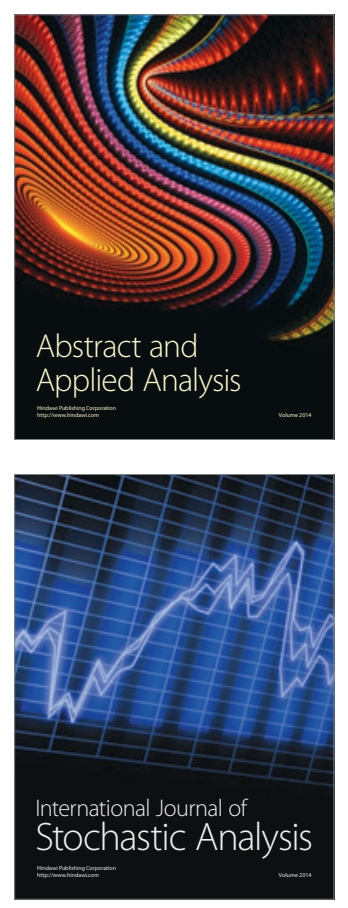

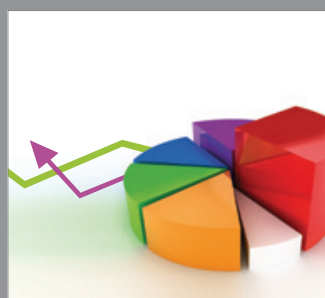

ournal of

Probability and Statistics

Promensencen
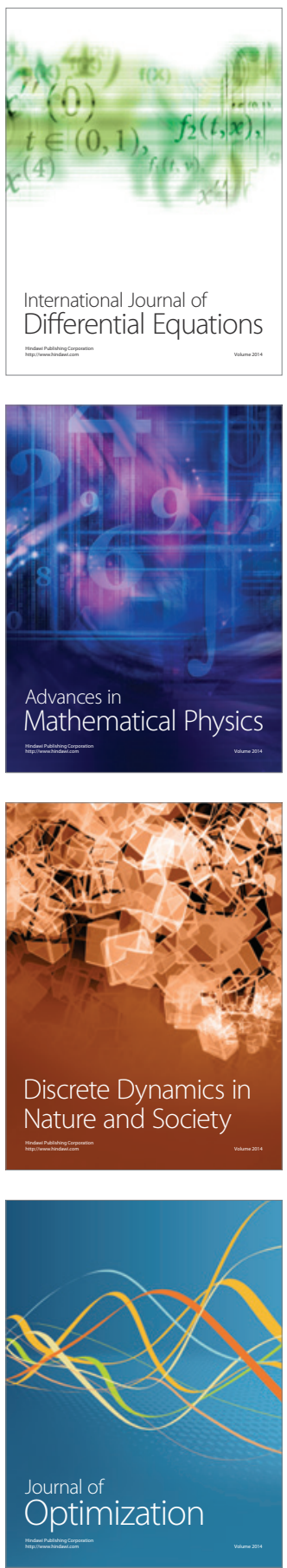